\title{
Equilibrium current and orbital magnetization in a quantum Hall fluid
}

\author{
Michael R. Geller and G. Vignale \\ Department of Physics, University of Missouri, Columbia, Missouri 65211
}

(June 21, 2021)

\begin{abstract}
We present a general theory for the equilibrium current distribution in an interacting two-dimensional electron gas subjected to a perpendicular magnetic field, and confined by a potential that varies slowly on the scale of the magnetic length. The distribution is found to consist of strips or channels of current, which alternate in direction, and which have universal integrated strength.
\end{abstract}

Typeset using REVTEX 


\section{Introduction}

The quantum Hall effect [可] is described as the quantization of the off-diagonal conductance of a two-dimensional electronic device, at integral or simple fractional multiples of $e^{2} / h$, and the vanishing of the diagonal conductance, when a perpendicular magnetic field of appropriate strength is present. It has long been understood [2] that this "appropriate strength" of the magnetic field is such that the the Fermi level lies in a gap of the spectrum of bulk extended states. Finer features of the effect are not nearly as well understood. There is considerable confusion, for instance, concerning the question of the physical distribution of the Hall current in a quantum Hall device. Is the Hall current carried by "edge states" residing near the the edges of the device, or is it carried by the bulk of the electron fluid? While early work [3], based on a model of non-interacting electrons, supported the first alternative, more recent work [四] taking into account Coulomb interactions self-consistently, concludes that at least a substantial part of the current is carried in the bulk. Experimental attempts to resolve the ambiguity by directly imaging the voltage and/or current distribution, have been made [5], with no conclusive results so far. A major practical difficulty is that in a nonuniform electron fluid subjected to a magnetic field, a finite current density exists even in thermal equilibrium, due to the time-reversal symmetry breaking action of the magnetic field. Although the net current through the device is zero, its local value may be quite large - in fact larger than the Hall current density, defined as the difference between the total current density measured in the transport regime and the current density at equilibrium. It is evident that a clear understanding of the properties of the equilibrium current distribution is a prerequisite for the understanding of experiments on the Hall current distribution. In two recent papers [6,7] we studied in detail the properties of this distribution, and found that they could be summarized in a few fairly general statements. We considered a confining potential that varies slowly on the scale of the magnetic length $l: V^{\prime} l<<\Delta$ where $\Delta$ is the minimum energy gap that we want to to take into account $\left(\Delta=\hbar \omega_{c}\right.$ if the fractional quantum Hall effect is ignored). This condition - at least insofar as the integral Hall effect is concerneed - is very well satisfied in realistic devices, in which the confinement lengths 
are of the order of $10^{4} \AA$ or more, while $l$ is of the order of $100 \AA$ [ 8 . Our expression, which correctly describes the current distribution on a scale larger than $l$, has two components: an "edge" current, which is proportional to the density gradient, and a "bulk" current, which is proportional to the gradient of a self-consistent potential. In the limit of zero temperature these two contributions become separate in space: The edge current flows in the "compressible" regions, in which the density varies, while the self-consistent potential remains constant. The bulk current flows in the "incompressible" regions, where the self-consistent potential varies and the density is constant. The directions of these two types of currents generally display a striking alternating pattern. Furthermore, the integrated currents across a compressible or incompressible region are universal, in the sense of depending only on the chemical potentials of a uniform two-dimensional electron gas near an incompressible state.

\section{Current Density Distribution}

A convenient tool for the study of equilibrium orbital currents in an interacting electronic system is provided by the current-density functional theory (CDFT) [9]. This formulation provides a rigorous mapping of the many-body problem for the ground-state energy, density, and current density, to an effective one-body problem, in which independent electrons move under the action of self-consistent local scalar and vector potentials. Unlike the ordinary density functional theory, CDFT is rigorous, in principle, for the calculation of equilibrium currents. The effective single particle equations have the form 9]

$$
\begin{aligned}
\left(\frac{1}{2 m}[-i \vec{\nabla}+\right. & \left.\frac{e}{c} \vec{A}(\vec{r})\right]^{2}+V(\vec{r})+V_{H}(\vec{r})+V_{x c}(\vec{r})+\left[-i \vec{\nabla}+\frac{e}{c} \vec{A}(\vec{r})\right] \cdot \frac{e}{2 m c} \vec{A}_{x c}(\vec{r}) \\
& \left.+\frac{e}{2 m c} \vec{A}_{x c}(\vec{r}) \cdot\left[-i \vec{\nabla}+\frac{e}{c} \vec{A}(\vec{r})\right]-\frac{e}{c} \frac{\vec{j}(\vec{r}) \cdot \vec{A}_{x c}(\vec{r})}{n(\vec{r})}\right) \psi_{\alpha}(\vec{r})=\epsilon_{\alpha} \psi_{\alpha}(\vec{r}),
\end{aligned}
$$

where $\vec{A}(\vec{r})$ and $V(\vec{r})$ are the external vector and scalar potentials, $n(\vec{r})$ is the number density, and $\vec{j}(\vec{r})$ is the current density. Here

$$
V_{H}(\vec{r})=e^{2} \int d \vec{r}^{\prime} \frac{n\left(\vec{r}^{\prime}\right)}{\left|\vec{r}-\vec{r}^{\prime}\right|}
$$

is the Hartree potential, 


$$
V_{x c}(\vec{r})=\frac{\delta E_{x c}\left[n(\vec{r}), B_{\nu}(\vec{r})\right]}{\delta n(\vec{r})}
$$

is the exchange-correlation (xc) scalar potential, and

$$
\frac{e}{c} \vec{A}_{x c}(\vec{r})=-\frac{m c}{e n(\vec{r})} \vec{\nabla} \times \frac{\delta E_{x c}\left[n(\vec{r}), B_{\nu}(\vec{r})\right]}{\delta \vec{B}_{\nu}(\vec{r})}
$$

is the exchange-correlation vector potential. A crucial feature of the theory is that the xc energy functional $E_{x c}\left[n(\vec{r}), B_{\nu}(\vec{r})\right]$ is a functional of the density $n(\vec{r})$ and the vorticity 9

$$
\vec{B}_{\nu}(\vec{r})=\vec{B}(\vec{r})-\frac{m c}{e} \vec{\nabla} \times\left(\frac{\vec{j}(\vec{r})}{n(\vec{r})}\right),
$$

where $\vec{B}(\vec{r})=\vec{\nabla} \times \vec{A}(\vec{r})$ is the magnetic field. The density and orbital current density are self-consistently determined by sums of orbitals, which are solutions of eq. (11). We have ignored spin for simplicity. The theory would yield the exact density and current distributions, if the exact exchange-correlation energy functional were known. In the local density approximation (LDA), which is justified for density distributions that are slowly varying on the scale of the magnetic length, $E_{x c}$ takes the form

$$
E_{x c}\left[n(\vec{r}), B_{\nu}(\vec{r})\right] \simeq \int \epsilon_{x c}\left[n(\vec{r}), B_{\nu}(\vec{r})\right] d \vec{r}
$$

where $\epsilon_{x c}[n, B]$ is the xc energy density of the two-dimensional electron gas (2DEG) of uniform density $n$ in a uniform magnetic field $B$.

We now specialize to the case of a uniform magnetic field perpendicular to the plane of the electrons $\vec{A}(\vec{r})=B x \hat{y}$ in the Landau gauge, and a slowly varying confining potential $V(\vec{r})$. Following the approach of [6], at each point $\vec{r}_{0}$ in space we define local cartesian axes such that the $x$ axis is along the direction of the gradient of the total self-consistent potential $V_{H x c} \equiv V+V_{H}+V_{x c}$. Thus, the total potential is locally a function of the $x$ coordinate only. Choosing also the Landau gauge in such a way that $\vec{A}$ depends only on the local $x$ coordinate (and is parallel to the local $y$ axis) we effectively obtain a local one-dimensional problem. In particular, the density and current profiles depend only on the local $x$ coordinate. This is so, because an $x$-dependent density and current profile leads to an $x$-dependent xc vector potential in the $y$ direction given by the formula 


$$
\frac{e}{c} A_{x c}(x)=-\frac{m c}{e n(x)} \frac{d}{d x} \frac{\delta E_{x c}\left[n(x), B_{\nu}(x)\right]}{\delta B_{\nu}(x)}
$$

It is then clear that the eigenfunctions of the Kohn-Sham equation localized near $\vec{r}_{0}$ have the form

$$
\psi_{n k}(x, y)=\frac{1}{\sqrt{L}_{y}} e^{i k y} \psi_{n k}(x)
$$

where the $\psi_{n k}(x)$ are normalized solutions of the equation

$$
\left(-\frac{1}{2} \frac{d^{2}}{d x^{2}}+\frac{1}{2}(x-X)^{2}+V_{H x c}(x)+\frac{e}{c} A_{x c}(x)\left[x-X-\frac{j_{y}(x)}{n(x)}\right]\right) \psi_{n k}(x)=\epsilon_{n X} \psi_{n k}(x),
$$

where $x$ is in units of $l, n$ is in units of $l^{-2}, j_{y}$ is in units of $\hbar / 2 m l^{3}, V_{H x c}$ is in units of $\hbar \omega_{c}, e A_{x c} / c$ is in units of $\hbar / l$, and $X=-k l$ is the guiding center of the Landau orbital). A more rigorous discussion can be given in terms of exponentially localized Green's functions [6]. Eq. (9) may be solved perturbatively, which is exact in the limit of slowly varying potential. Because the eigenfunctions of $-d^{2} / d x^{2}+(x-X)^{2}$ are strongly localized about $x=X$ we can expand

$$
V_{H x c}(x) \approx V_{H x c}(X)+(x-X) V_{H x c}^{\prime}(X)
$$

and use

$$
\frac{e}{c} A_{x c}(x)\left[x-X-\frac{j_{y}(x)}{n(x)}\right] \approx \frac{e}{c} A_{x c}(X) \cdot[x-X]
$$

In writing down the second equation we have exploited two facts: (i) $A_{x c}(x)$ being given by eq.(7) is already a gradient of a local function of $x$. Hence, we truncate its expansion to the zeroth order in $x-X$ (ii) $j_{y}(x)$, as it will soon be clear, is also a gradient of a local function of $x$, therefore its multiplication by $A_{x c}(x)$ is the product of two gradients, which must be regarded as a higher order infinitesimal quantity and hence dropped in the present theory.

Using equations (10) and (11), equation (9) can be solved, and the density and current density can be computed explicitely. The general character of the density distribution has been discussed at length elsewhere [8,6,7]. Very briefly, the self-consistent density profile 
consists of alternating compressible and incompressible regions. A typical profile is shown by the solid line in Fig. 1. In a compressible region, the density varies while the selfconsistent potential is pinned to a constant value. In an incompressible region, the density is pinned to an integral or fractional multiple of $1 / 2 \pi l^{2}$, and the self-consistent potential varies. The self-consistent chemical potential is tied to a set of quasi-degenerate single particle orbitals, which are localized in the compressible regions. Rather than insisting on the properties of the density distribution, which are well understood, here we focus on the result for the current density, which can be stated as follows:

$$
j_{y}(x)=\gamma_{k} \frac{d n}{d x}+2 n(x)\left[V_{H x c}^{\prime}(x)+\frac{e}{c} A_{x c}(x)\right]
$$

where

$$
\gamma_{k}[n, B] \equiv(2[\nu]+1)
$$

and $[\nu]$ is the integral part of the filling factor. As a final step, we observe that from eq. (7), combined with the local density approximation, eq. (6), we obtain

$$
2 \frac{e}{c} A_{x c}(x) n(x)=\gamma_{x c}[n, B] \frac{d n(x)}{d x}
$$

where we have defined

$$
\gamma_{x c}[n, B] \equiv \frac{1}{\mu_{B}} \frac{\partial \mu_{x c}[n, B]}{\partial B} .
$$

Here $\mu_{B} \equiv e \hbar / 2 m c$ is the Bohr magneton and $\mu_{x c} \equiv \partial \epsilon_{x c}[n, B] / \partial n$ is the xc part of the chemical potential of the uniform 2DEG. We have set $B_{\nu}=B$ after taking the functional derivative of $E_{x c}$, since $A_{x c}$ is already of the desired order in the gradient of the density. It is important that, in the definition of $\gamma_{x c}[n, B]$, the density is never taken to be exactly equal to one of the incompressible densities at which $\mu_{x c}$ is discontinuous. This prescription assures that $\gamma_{x c}$ is finite everywhere, although possibly discontinuous at the incompressible densities.

Restoring the physical units and returning to the original coordinate system we finally write our result as 


$$
\vec{j}(\vec{r})=\frac{\hbar}{2 m}\left(\gamma_{k}[n, B]+\gamma_{x c}[n, B]\right) \vec{\nabla} n(\vec{r}) \times \hat{z}+\frac{1}{m \omega_{c}} n(\vec{r}) \vec{\nabla} V_{H x c}(\vec{r}) \times \hat{z}
$$

To understand this formula, we must keep in mind that, in the low temperature limit, the "bulk" term $(\vec{\nabla} V(\vec{r}))$ and the "edge" term $(\vec{\nabla} n(\vec{r}))$ contribute to mutually exclusive regions of space - incompressible and compressible regions, respectively. In the incompressible regions, the density is pinned to one of the quantized values, and the bulk term reduces to the usual Hall current $\vec{j}=\left(e \nu_{i} / h\right) \vec{\nabla} V_{H x c}(\vec{r}) \times \hat{z}$, where $\nu_{i}$ is a quantized filling factor, and $\vec{\nabla} V_{H x c}$ plays the role of the electric field. Assuming, for the sake of definiteness, that the self-consistent potential increases monotonically from the center to the perifery of a quantum Hall system (for example a bar or a dot), this contribution to the current is found to be paramagnetic. In the compressible regions, the self-consistent potential is pinned, while the density varies. Here the edge term becomes operative. Under the assumption that the density decreases monotonically from the center to the perifery of the system, its contribution to the current is found to be diamagnetic. A typical self-consistent current density profile is shown as a dashed line in Fig. 1.

\section{Orbital Magnetization}

Since the charge current $\vec{J}(\vec{r}) \equiv-e \vec{j}(\vec{r})$ satisfies the continuity equation

$$
\vec{\nabla} \cdot \vec{J}(\vec{r})=0
$$

it is possible to write it as the curl of a local function $M(\vec{r}) \hat{z}$ which we call the "orbital magnetization":

$$
\vec{J}(\vec{r})=c \vec{\nabla} \times M(\vec{r}) \hat{z}
$$

It is evident that $M(\vec{r})$ is defined only up to an arbitrary additive constant. However, in the limit of slowly varying density, the orbital magnetization may be derived from the internal energy of the uniform electron gas

$$
M(\vec{r})=-\frac{\partial \epsilon[n(\vec{r}), B]}{\partial B} .
$$

This gives [10] 


$$
\vec{J}(\vec{r})=-c \frac{\partial^{2} \epsilon[n, B]}{\partial n \partial B} \vec{\nabla} n \times \hat{z}
$$

with $\epsilon \equiv \epsilon_{k}+\epsilon_{x c}$ the total internal energy (kinetic plus exchange-correlation) per unit area of the uniform 2DEG. It is not immediately evident that eq.(20) is equivalent to eq.(16). A puzzling feature is that the $\vec{\nabla} V(\vec{r})$ term does not appear explicitly in eq. 20). The puzzle is resolved by noting that the thermodynamic derivative appearing in eq. (20) has singularities when the density is such that the compressibility vanishes. These singular contributions are responsible for the appearance of the $\vec{\nabla} V(\vec{r})$ term in the incompressible regions. To show this, we rewrite $\partial^{2} \epsilon / \partial n \partial B$ as

$$
\frac{\partial^{2} \epsilon[n, B]}{\partial n \partial B}=\left(\frac{\partial \mu}{\partial B}\right)_{n}=-\frac{(\partial n / \partial B)_{\mu}}{(\partial n / \partial \mu)_{B}} .
$$

When the density is not such that the compressibility vanishes, the derivatives are finite, and it is easy to verify that eq. (20) reduces to the first term of eq. (16). But when the density is such that the compressibility vanishes, we must turn to the second equality in (21), for insight into the behavior of the current. The denominator $(\partial n / \partial \mu)_{B}$ vanishes, because the system is incompressible. At the same time $\vec{\nabla} n(\vec{r})$ also vanishes in the regions where incompressibility holds. The ratio of these two quantities, however, remains finite in the limit $T \rightarrow 0$ :

$$
-\left(\frac{\partial \mu}{\partial n}\right)_{B} \vec{\nabla} n(\vec{r}) \rightarrow \vec{\nabla} V_{H x c}(\vec{r}) .
$$

The numerator $(\partial n / \partial B)_{\mu}$ is well known to be proportional to the Hall conductivity $e^{2} \nu_{i} / h$ [11. This establishes the equivalence of eqs. (20) and (16).

The usefulness of writing the current as the curl of the orbital magnetization lies in the fact that it makes possible to express the flux of current across an arbitrary path as the difference in the values of the orbital magnetization at the end points:

$$
I_{1,2}=\int_{1}^{2} \vec{j}(\vec{r}) \cdot \hat{n}(\vec{r}) d l=c[M(1)-M(2)]
$$

where the line integral is along a path joining points 1 and 2 , and $\hat{n}$ is the unit vector normal to the path. The key observation is that the orbital magnetization at the end points may be 
well represented by the LDA even if it is not well represented at intermediate points along the path.

As a simple example, consider the disk of non-interacting electrons studied recently by Avishai and Kohmoto [12 (AK). The integrated current across a radius is simply the magnetization at the center of the dot, since the magnetization obviously vanishes at the edge of the system. But the density is uniform at the center of the dot, so the LDA is certainly valid there. Furthermore, under the conditions envisaged by AK, the Fermi energy is pinned to the bottom of the i-th Landau level in the bulk, which means that $\mu=(i+1 / 2) \hbar \omega_{c}^{+}$ (a superscript "+" or "-" means that an infinitesimal positive quantity must be added or subtracted). Looking at the relation between $n$ and $\mu$ in the non-interacting 2DEG at an infinitesimal temperature we see that this chemical potential corresponds to a density $n=\left[(i+1) / 2 \pi l^{2}\right]^{-}$at the center of the dot. This, in turn, corresponds to an orbital magnetization $M=-\left(i \mu_{B} / 2 \pi l^{2}\right)$, as one can see from Fig. 2. Therefore the total current integrated from the center to the edge of the dot is equal to $-i e \omega_{c} / 4 \pi$, i.e., the total current is quantized in integral multiples of $e \omega_{c} / 4 \pi$. This result was first derived by AK from a numerical solution of the Schrödinger equation with hard wall boundary conditions. The present derivation demonstrates the generality of the result.

Next we consider the interacting electron fluid. The energy of the uniform phase consists of kinetic and exchange-correlation contributions. The kinetic energy is known exactly, and the kinetic contribution to the orbital magnetization is given by

$$
M_{k}(\nu, B)=\left([\nu]-(2[\nu]+1)(\nu-[\nu]) \frac{\mu_{B}}{2 \pi l^{2}}\right.
$$

This curve is plotted in Fig. 2. No simple expression is known which represents $\epsilon_{x c}$ accurately at all values of filling factor $\nu$. However, to calculate the integrated current in a compressible or incompressible region, we only need to know what happens in the vicinity of an incompressible filling factor $\nu_{0}$, integral or fractional. Here some essentially exact results can be obtained. In a neighborhood of $\nu_{0}$ the exchange-correlation energy density can be expanded as follows: 


$$
\epsilon_{x c}(\nu, B)=\left[\epsilon_{x c 0}+\frac{\left(\nu-\nu_{0}\right)}{2 \pi l^{2}} \mu_{x c}\left[\nu_{0}^{+}, B\right]+O\left(\left(\nu-\nu_{0}\right)^{3 / 2}\right)\right]
$$

for $\nu>\nu_{0}$, and

$$
\epsilon_{x c}(\nu, B)=\left[\epsilon_{x c 0}+\frac{\left(\nu-\nu_{0}\right)}{2 \pi l^{2}} \mu_{x c}\left[\nu_{0}^{-}, B\right]+O\left(\left(\nu_{0}-\nu\right)^{3 / 2}\right)\right]
$$

for $\nu<\nu_{0}$. Here $\epsilon_{x c 0}$ is the xc energy density of the incompressible state at filling factor $\nu_{0}$ and $\mu_{x c}\left[\nu_{0}^{+}, B\right]$ and $\mu_{x c}\left[\nu_{0}^{-}, B\right]$ are the xc chemical potentials calculated as right or left derivatives of the xc energy density with respect to density. In the limit of high magnetic field $\left(e^{2} / l \hbar \omega_{c}<<1\right) \epsilon_{x c 0}$ scales as $\epsilon_{x c 0}(B)=\bar{\epsilon}_{x c 0} e^{2} / 2 \pi l^{3}$ and the $\mu_{x c}$ 's scale as $\mu_{x c}(\nu, B)=$ $\bar{\mu}_{x c}(\nu) e^{2} / l$, where the dimensionless quantities $\bar{\epsilon}_{x c 0}$ and $\bar{\mu}_{x c}(\nu)$ are independent of magnetic field. The $O\left(\left(\nu-\nu_{0}\right)^{3 / 2}\right)$ term on the right hand side represents the interaction energy of a classical Wigner crystal of dilute quasiparticles (or quasiholes) added to the incompressible state. It is easy to verify that this term - as well as all the higher order terms in the expansion of the energy in $\nu-\nu_{0}$ - does not contribute to the orbital magnetization in the limit $\nu \rightarrow \nu_{0}^{ \pm}$. Taking the derivatives of eq. (25) and eq. (26) with respect to $B$, using the power law scaling of the energy and chemical potentials as functions of $B$, we obtain the exchange-correlation contribution to the magnetization

$$
M_{x c}\left(\nu=\nu_{o}^{ \pm}, B\right)=\left[-3 \bar{\epsilon}_{x c 0}+2 \nu_{0} \bar{\mu}_{x c}\left(\nu_{0}^{ \pm}\right)\right] \frac{e^{2}}{l \hbar \omega_{c}} \frac{\mu_{B}}{2 \pi l^{2}}
$$

The above equation may be used to calculate the exact total current that flows through a compressible strip or channel connecting an incompressible region at filling factor $\nu_{0}$ to another incompressible region at filling factor $\nu_{1}$. Assuming, for definiteness, that $\nu_{0}>\nu_{1}$ and integrating the current flow along a line going from the higher to the lower density we find that the total current is

$$
I=c\left[M\left(\nu=\nu_{0}^{-}, B\right)-M\left(\nu=\nu_{1}^{+}, B\right)\right],
$$

where $M=M_{k}+M_{x c}$. The remarkable feature of this result is that it holds true despite the lack of detailed information about the density and current distribution within the compressible region, i.e., despite the fact that $n(\vec{r})$ and $\gamma_{x c}(n, B)$ are not known everywhere inside 
the region. Equation (27) can also be used to calculate the current flowing through the incompressible region, say at filling factor $\nu_{1}$. For this region we obtain

$$
I=c\left[M\left(\nu_{1}^{+}\right)-M\left(\nu_{1}^{-}\right)\right]=\frac{e \nu_{1}}{h} \Delta_{1}
$$

where $\Delta_{1}=\mu\left(\nu_{1}^{+}, B\right)-\mu\left(\nu_{1}^{-}, B\right)$ is the gap in the electron chemical potential at filling factor $\nu_{1}$. This result is precisely what one would expect, based on the observation that the change in the self-consistent potential across an incompressible strip must equal the incompressibility gap.

\section{Conclusions}

In conclusion, we have elucidated in this paper the nature of the equilibrium current distribution in a two-dimensional electron gas confined by a potential which varies slowly on the

scale of the magnetic length. We have shown that the density profile consists of alternating compressible and incompressible regions, and that the current flows in opposite directions in these two types of regions, reflecting the sawtooth behavior of the magnetization of the uniform interacting electron gas as a function of density. Finally, we have demonstrated that the integrated current across a compressible or incompressible region can be expressed (in the limit of strong magnetic field) in terms of the chemical potentials of the uniform electron gas near incompressible filling factors. Detailed numerical results for exchange-correlation chemical potentials near integral filling factors will be reported elesewhere.

Acknowledgements We acknowledge support from NSF grant No. DMR-9403908. 


\section{REFERENCES}

[1] K. von Klitzing, G. Dorda, M. Pepper, Phys. Rev. Lett. 45, 494 (1980).

[2] The Quantum Hall Effect, edited by R. E. Prange and S. M. Girvin, (Springer, New York 1987).

[3] B. I. Halperin, Phys. Rev. B 25, 2185 (1982); A. H. MacDonald, T. M. Rice and W. F. Brinkman, Phys. Rev. B28, 3648 (1983).

[4] D. J. Thouless, Phys. Rev. Lett. 71, 1879 (1993).

[5] A. J. Kent et al., Helv. Physica Acta 65, 331 (1992); R. Knott et al., to be published.

[6] Michael R. Geller and G. Vignale, Phys. Rev. B 50, 11714 (1994).

[7] Michael R. Geller and G. Vignale, to be published (cond-mat/9412028).

[8] D. B. Chklovskii, B. I. Shklovskii, and L. I. Glazman, Phys. Rev. B 46, 4026 (1992).

[9] G. Vignale and M. Rasolt, Phys. Rev. B 37, 10685 (1988); G. Vignale, M. Rasolt, and D. J. W. Geldart, Adv. Quantum Chemistry 21, 235 (1990); G. Vignale in Proceedings of the NATO ASI on Density Functional Theory, Il Ciocco, Italy, 1993, edited by R. M. Dreizler and E. K. U. Gross, to be published.

[10] This expression was first derived in G. Vignale and P. Skudlarski, Phys. Rev. B46, 10232 (1992).

[11] P. Streda, Journal of Physics C: Solid State Physics 15, L717 (1982); 15, L1299 (1982).

[12] Y. Avishai, and M. Kohmoto, Phys. Rev. Lett. 71, 279 (1993). 


\section{FIGURES}

FIG. 1. Typical self-consistent equilibrium density (solid curve) and current density (dashed curve) for a two dimensional electron fluid confined along the $x$ direction at low temperature. The density is plotted in units of $\rho_{0}=1 / 2 \pi l^{2}$ and the current is plotted in units of $j_{0}=e \omega_{c} / 2 \pi l$. Note the alternating signs of the edge and bulk currents

FIG. 2. Orbital magnetization density versus filling factor for a non-interacting uniform two-dimensional electron gas at low temperature. 